\title{
Time fractional equations and probabilistic representation
}

\author{
(In Chaos, Solitons and Fractals 102 (2017), 168-174.)
}

\section{Zhen-Qing Chen}

January 12, 2019

\begin{abstract}
In this paper, we study the existence and uniqueness of solutions for general fractionaltime parabolic equations of mixture type, and their probabilistic representations in terms of the corresponding inverse subordinators with or without drifts. An explicit relation between occupation measure for Markov processes time-changed by inverse subordinator in open sets and that of the original Markov process in the open set is also given.
\end{abstract}

AMS 2010 Mathematics Subject Classification: Primary 26A33, 60H30; Secondary: 34K37

Keywords and phrases: fractional-time derivative, subordinator, inverse subordinator, Lévy measure, occupation measure

\section{Introduction}

Fractional calculus has attracted lots of attentions in several fields including mathematics, physics, chemistry, engineering, hydrology and even finance and social sciences (see [9, 20, 22, 21]). The classical heat equation $\partial_{t} u=\Delta u$ describes heat propagation in homogeneous medium. The timefractional diffusion equation $\partial_{t}^{\beta} u=\Delta u$ with $0<\beta<1$ has been widely used to model the anomalous diffusions exhibiting subdiffusive behavior, due to particle sticking and trapping phenomena (see e.g. [20, 23]). Here the fractional-time derivative $\partial_{t}^{\beta}$ is the Caputo derivative of order $\beta \in(0,1)$, which can be defined by

$$
\partial_{t}^{\beta} f(t)=\frac{1}{\Gamma(1-\beta)} \frac{d}{d t} \int_{0}^{t}(t-s)^{-\beta}(f(s)-f(0)) d s,
$$

where $\Gamma(\lambda):=\int_{0}^{\infty} t^{\lambda-1} e^{-t} d t$ is the Gamma function. The above definition says that the fractional derivative of $f$ at time $t$ depends on the whole history of $f(s)$ on $(0, t)$ with the nearest past affecting the present more. Meerschaert and Scheffer [17, Theorem 5.1] recognized, based on Baeumer and Meerschaert [2], that the solution to $u=u(t, x)$ of $\partial_{t}^{\beta} u=\Delta u$ with $u(0, x)=f(x)$ admits an interesting probabilistic representation:

$$
u(t, x)=\mathbb{E}_{x}\left[f\left(X_{E_{t}}\right)\right], \quad x \in \mathbb{R}^{d},
$$


where $X$ is Brownian motion on $\mathbb{R}^{d}$ with infinitesimal generator $\Delta$ and $E_{t}$ is an inverse $\beta$-stable subordinator that is independent of $X$. In fact, the above representation was proved for a large class of operators $\mathcal{L}$ in place of $\Delta$ that generates a strong Markov process $X$. This representation connects probability theory to time fractional equations. The scaling property of the $\beta$-stable subordinator is used in a crucial way in their derivation.

In applications and numerical approximations [8], there is a need to consider generalized fractionaltime derivatives where its value at time $t$ may depend only on the finite range of the past from $t-\delta$ to $t$, for example, $\frac{d}{d t} \int_{(t-\delta)^{+}}^{t}(t-s)^{-\beta}(f(s)-f(0)) d s$. Here for $a \in \mathbb{R}, a^{+}:=\max \{a, 0\}$. Motivated by this, for a given function $w:(0, \infty) \rightarrow[0, \infty)$ that is locally integrable on $[0, \infty)$, we introduce a generalized fractional-time derivative

$$
\partial_{t}^{w} f(t)=\frac{d}{d t} \int_{0}^{t} w(t-s)(f(s)-f(0)) d s
$$

whenever it is well defined. Typically $w(t)$ is a non-negative decreasing function on $(0, \infty)$ that blows up at $t=0$. Clearly, when $w(s)=\frac{1}{\Gamma(1-\beta)} s^{-\beta}$ for $\beta \in(0,1), \partial_{t}^{w} f$ is just the Caputo derivative of order $\beta$ defined by (1.1).

Let $X=\left\{X_{t}, t \geq 0 ; \mathbb{P}_{x}, x \in E\right\}$ be a strong Markov process on a separable locally compact Hausdorff space $E$ whose transition semigroup $\left\{P_{t}, t \geq 0\right\}$ is a uniformly bounded strong continuous semigroup in some Banach space $(\mathbb{B},\|\cdot\|)$. For example, $\mathbb{B}=L^{p}(E ; m)$ for some measure $m$ on $E$ and $p \geq 1$ or $\mathbb{B}=C_{\infty}(E)$, the space of continuous functions on $E$ that vanish at infinity equipped with uniform norm. Let $(\mathcal{L}, \mathcal{D}(\mathcal{L}))$ be the infinitesimal generator of $\left\{P_{t}, t \geq 0\right\}$ in $\mathbb{B}$. In this paper, we are interested in the existence and uniqueness of solution $u=u(t, x)$ for

$$
\kappa \frac{\partial u}{\partial t}+\partial_{t}^{w} u=\mathcal{L} u \quad \text { with } u(0, x)=f(x)
$$

and its probabilistic representation, where $\kappa \geq 0$ is a positive constant. We will also address the following question: given a subordinator $S=\left\{S_{t} ; t \geq 0\right\}$ that is independent of $X$, what equation does $u(t, x):=\mathbb{E}_{x}\left[f\left(X_{E_{t}}\right)\right]$ satisfy?

Given a constant $\kappa \geq 0$ and an unbounded right continuous non-increasing function $w(x)$ on $(0, \infty)$ with $\lim _{x \rightarrow \infty} w(x)=0$ and $\int_{0}^{\infty}(1 \wedge x)(-d w(x))<\infty$, there is a unique non-negative valued Lévy process $\left\{S_{t} ; t \geq 0\right\}$ with $S_{0}=0$ (called subordinator) associated with it in the following way. Here for $a, b \in \mathbb{R}, a \wedge b:=\min \{a, b\}$. Let $\mu$ be the measure on $(0, \infty)$ so that $w(x)=\mu(x, \infty)$. Clearly

$$
\mu(0, \infty)=\infty \text { and } \quad \int_{0}^{\infty}(1 \wedge x) \mu(d x)<\infty .
$$

It is well-known (cf. 3]) that there is subordinator $\left\{S_{t} ; t \geq 0\right\}$ with Laplace exponent $\phi$ :

$$
\mathbb{E}\left[e^{-\lambda S_{t}}\right]=e^{-t \phi(\lambda)}, \quad \lambda>0,
$$

so that

$$
\phi(\lambda)=\kappa \lambda+\int_{0}^{\infty}\left(1-e^{-\lambda x}\right) \mu(d x) .
$$

The measure $\mu$ is called the Lévy measure of the subordinator.

Conversely, given a subordinator $\left\{S_{t} ; t \geq 0\right\}$, there is a unique constant $\kappa \geq 0$ and a Lévy measure $\mu$ on $(0, \infty)$ satisfying $\int_{0}^{\infty}(1 \wedge x) \mu(d x)<\infty$ so that (1.3) and (1.4) hold. Throughout this 
paper, $\left\{S_{t} ; t \geq 0\right\}$ is such a general subordinator with infinite Lévy measure $\mu$ and possibly with drift $\kappa \geq 0$. When $\kappa=0$, we say the subordinator is driftless or with no drift. Define for $t>0$, $E_{t}=\inf \left\{s>0: S_{s}>t\right\}$, the inverse subordinator. The assumption that the Lévy measure $\mu$ is infinite (which is equivalent to $w(x):=\mu(x, \infty)$ being unbounded) excludes compounded Poisson processes. Under this assumption, almost surely, $t \mapsto S_{t}$ is strictly increasing and hence $t \mapsto E_{t}$ is continuous.

The main purpose of this paper is to establish the following.

Theorem 1.1 Under the above setting, let $w(x)=\mu(x, \infty)$, which is an unbounded right continuous non-increasing function on $(0, \infty)$. The function $u(t, x):=\mathbb{E}_{x}\left[f\left(X_{E_{t}}\right)\right]$ is the unique solution in $\mathbb{B}$ to the time fractional equation

$$
\left(\kappa \partial_{t}+\partial_{t}^{w}\right) u=\mathcal{L} u \quad \text { with } u(0, x)=f(x)
$$

in the strong sense (see Theorem 2.3 for a precise statemnt) for every $f \in \mathcal{D}(\mathcal{L})$. Here $\partial_{t}$ is the time derivative $\frac{\partial}{\partial t}$.

Our method of proof to the above theorem is different from that of [2] which is for stable subordinators, as there is no scaling property for a general subordinator $S_{t}$. Our approach is quite robust and direct that works for any subordinator with infinite Lévy measure and for a wide class of infinitesimal generators. One feature of this paper is that possible mixture of the standard time derivative $\partial_{t}$ and the general fractional time derivative $\partial_{t}^{w}$ is covered and treated in a unified way. Moreover, we will establish a more general result for $\mathcal{L}$ being the infinitesimal generator of any uniformly bounded strongly continuous semigroup in general Banach spaces; see Theorem 2.3 for a precise statement. Our Theorem 2.3 not only gives the existence but also the uniqueness of solutions to the time fractional equation. The generalized Caputo derivative defined by (1.2) with $w(x)=\mu(x, \infty)$ extends the distributed order fractional derivative defined in [18] where $S_{t}$ is a mixture of $\beta$-stable subordinators. An important application of these more general time fractional derivatives is to model "ultraslow diffusion" where a plume spreads at a logarithmic rate; see [18] for details.

In Section 3 of this paper, we will study the relation between occupation measure for the timechanged process $X^{*}:=X_{E_{t}}$ by inverse subordinator in an open set $D \subset E$ with that of $X$ in $D$.

\section{General time fractional equations}

Recall that $\left\{S_{t} ; t \geq 0\right\}$ is a general subordinator with infinite Lévy measure $\mu$ and drift $\kappa \geq 0$, whose Laplace exponent $\phi(\lambda)$ is given by (1.4). Define $w(x)=\mu(x, \infty)$ for $x>0$ and $\phi_{0}(\lambda):=$ $\int_{0}^{\infty}\left(1-e^{-\lambda x}\right) \mu(d x)$. Note that $\phi_{0}(\lambda)$ is the Laplace exponent of the driftless subordinator $\left\{\bar{S}_{t}:=\right.$ $\left.S_{t}-\kappa t, t \geq 0\right\}$ having Lévy measure $\mu$. Clearly

$$
\phi(\lambda)=\kappa \lambda+\phi_{0}(\lambda) \quad \text { and } \quad S_{t}=\kappa t+\bar{S}_{t} .
$$

Since $\mu(0, \infty)=\infty$, almost surely, $t \mapsto \bar{S}_{t}$ is strictly increasing. 
For every $a>0$, by Fubini theorem,

$$
\int_{0}^{a} w(x) d x=\int_{0}^{a}\left(\int_{(x, \infty)} \mu(d \xi)\right) d x=\int_{0}^{\infty}\left(\int_{0}^{\xi \wedge a} d x\right) \mu(d \xi)=\int_{0}^{\infty}(\xi \wedge a) \mu(d \xi)<\infty .
$$

The Laplace transform of $w$ is

$$
\begin{aligned}
\int_{0}^{\infty} e^{-\lambda x} w(x) d x & =\int_{0}^{\infty} e^{-\lambda x} \int_{(x, \infty)} \mu(d \xi) d x=\int_{0}^{\infty}\left(\int_{0}^{\xi} e^{-\lambda x} d x\right) \mu(d \xi) \\
& =\frac{1}{\lambda} \int_{0}^{\infty}\left(1-e^{-\lambda \xi}\right) \mu(d \xi)=\frac{\phi_{0}(\lambda)}{\lambda}
\end{aligned}
$$

Lemma 2.1 There is a Borel set $\mathcal{N} \subset(0, \infty)$ having zero Lebesgue measure so that

$$
\mathbb{P}\left(\bar{S}_{s} \geq t\right)=\int_{0}^{s} \mathbb{E}\left[w\left(t-\bar{S}_{r}\right) 1_{\left\{t \geq \bar{S}_{r}\right\}}\right] d r \quad \text { for every } s>0 \text { and } t \in(0, \infty) \backslash \mathcal{N}
$$

Consequently, for every $t \in(0, \infty) \backslash \mathcal{N}, s \mapsto \mathbb{P}\left(\bar{S}_{s} \geq t\right)$ is continuous and $\mathbb{P}\left(\bar{S}_{s}=t\right)=0$ for every $s>0$.

Proof. Note that since $r \mapsto \bar{S}_{r}$ is strictly increasing a.s., by Fubini theorem,

$$
\int_{0}^{s} \mathbb{E}\left[w\left(t-\bar{S}_{r}\right) 1_{\left\{t \geq \bar{S}_{r}\right\}}\right] d r=\int_{0}^{s} \mathbb{E}\left[w\left(t-\bar{S}_{r}\right) 1_{\left\{t>\bar{S}_{r}\right\}}\right] d r .
$$

For each fixed $s>0$, the Laplace transform of $t \mapsto \mathbb{P}\left(\bar{S}_{s} \geq t\right)$ is

$$
\begin{aligned}
\int_{0}^{\infty} e^{-\lambda t} \mathbb{P}\left(\bar{S}_{s} \geq t\right) d t & =\int_{0}^{\infty} e^{-\lambda t} \mathbb{P}\left(\bar{S}_{s}>t\right) d t \\
& =-\frac{1}{\lambda} \int_{0}^{\infty} \mathbb{P}\left(\bar{S}_{s}>t\right) d e^{-\lambda t}=\frac{1}{\lambda}+\frac{1}{\lambda} \int_{0}^{\infty} e^{-\lambda t} d_{t} \mathbb{P}\left(\bar{S}_{s}>t\right) \\
& =\frac{1}{\lambda}-\frac{1}{\lambda} \mathbb{E}\left[e^{-\lambda \bar{S}_{s}}\right]=\frac{1-e^{-s \phi_{0}(\lambda)}}{\lambda}
\end{aligned}
$$

By Fubini theorem and (2.3) , the Laplace transform of $t \mapsto \int_{0}^{s} \mathbb{E}\left[w\left(t-\bar{S}_{r}\right) 1_{\left\{t \geq \bar{S}_{r}\right\}}\right] d r$ is

$$
\begin{aligned}
\int_{0}^{\infty} e^{-\lambda t}\left(\int_{0}^{s} \mathbb{E}\left[w\left(t-\bar{S}_{r}\right) 1_{\left\{t \geq \bar{S}_{r}\right\}}\right] d r\right) d t & =\int_{0}^{s} \mathbb{E}\left[\int_{0}^{\infty} e^{-\lambda t} w\left(t-\bar{S}_{r}\right) 1_{\left\{t>\bar{S}_{r}\right\}} d t\right] d r \\
& =\int_{0}^{s} \mathbb{E}\left[e^{-\lambda \bar{S}_{r}} \int_{0}^{\infty} e^{-\lambda x} w(x) d x\right] d r \\
& =\frac{\phi_{0}(\lambda)}{\lambda} \int_{0}^{s} e^{-r \phi_{0}(\lambda)} d r=\frac{1-e^{-s \phi_{0}(\lambda)}}{\lambda},
\end{aligned}
$$

which is the same as the Laplace transform of $t \mapsto \mathbb{P}\left(\bar{S}_{s}>t\right)$. By the uniqueness of the Laplace transform that for each fixed $s>0$,

$$
\mathbb{P}\left(\bar{S}_{s} \geq t\right)=\int_{0}^{s} \mathbb{E}\left[w\left(t-\bar{S}_{r}\right) 1_{\left\{t \geq \bar{S}_{r}\right\}}\right] d r
$$


for a.e. $t>0$. Hence there is a Borel subset $\mathcal{N} \subset(0, \infty)$ having zero Lebesgue measure so that (2.4) holds for every $t \in(0, \infty) \backslash \mathcal{N}$ and for every rational $s>0$. Note that for each fixed $t>0, s \mapsto \mathbb{P}\left(\bar{S}_{s} \geq\right.$ $t)$ is right-continuous. On the other hand, for each fixed $t>0, s \mapsto \int_{0}^{s} \mathbb{E}\left[w\left(t-\bar{S}_{r}\right) 1_{\left\{t \geq \bar{S}_{r}\right\}}\right] d r$ is continuous. It follows that (2.4) holds for every $t \in(0, \infty) \backslash \mathcal{N}$ and every $s>0$. Consequently, for every $t \in(0, \infty) \backslash \mathcal{N}, s \mapsto \mathbb{P}\left(\bar{S}_{s} \geq t\right)$ is continuous. Since the subordinator $t \mapsto \bar{S}_{t}$ is strictly increasing a.s. and is stochastically continuous in the sense that $\mathbb{P}\left(\bar{S}_{r}=\bar{S}_{r-}\right)=1$ for all $r>0$, we have

$$
\mathbb{P}\left(\bar{S}_{s} \geq t\right)=\lim _{r \uparrow s} \mathbb{P}\left(\bar{S}_{r} \geq t\right)=\mathbb{P}\left(\bar{S}_{s}>t\right) \quad \text { for every } s>0 .
$$

In other words, $\mathbb{P}\left(\bar{S}_{s}=t\right)=0$ for every $t \in(0, \infty) \backslash \mathcal{N}$ and all $s>0$.

Define $G(0)=0$ and $G(x)=\int_{0}^{x} w(t) d t$ for $x>0$. Then by (2.2),$G(x)$ is a continuous function on $[0, \infty)$ with $G^{\prime}(x)=w(x)$ on $(0, \infty)$. By the integration by parts formula, for every $t>0$,

$$
\begin{aligned}
\int_{0}^{t} w(t-r) \mathbb{P}\left(S_{s}>r\right) d r & =-\int_{0}^{t} \mathbb{P}\left(S_{s}>r\right) d_{r} G(t-r) \\
& =G(t)+\int_{0}^{t} G(t-r) d_{r} \mathbb{P}\left(S_{s}>r\right) \\
& =G(t)-\int_{0}^{t} G(t-r) d_{r} \mathbb{P}\left(S_{s} \leq r\right) \\
& =G(t)-\mathbb{E}\left[G\left(t-S_{s}\right) 1_{\left\{t \geq S_{s}\right\}}\right] .
\end{aligned}
$$

In particular,

$$
\mathbb{E}\left[G\left(t-S_{s}\right) 1_{\left\{t \geq S_{s}\right\}}\right] \leq G(t) \text { for every } t>0 .
$$

For each fixed $t>0$, by (2.2) and dominated convergence theorem,

$$
s \mapsto \int_{0}^{t} w(t-r) \mathbb{P}\left(S_{s}>r\right) d r=\int_{0}^{t} w(t-r) \mathbb{P}\left(S_{s} \geq r\right) d r
$$

is a right continuous increasing function. Hence by $(\underline{2.5}), s \mapsto \mathbb{E}\left[G\left(t-S_{s}\right) 1_{\left\{t \geq S_{s}\right\}}\right]$ is a right continuous decreasing function on $[0, \infty)$.

Corollary 2.2 Let $\mathcal{N} \subset(0, \infty)$ be the set in Lemma 2.1, which has zero Lebesgue measure.

(i) $\int_{0}^{\infty} \mathbb{E}\left[w\left(t-\bar{S}_{r}\right) 1_{\left\{t \geq \bar{S}_{r}\right\}}\right] d r=1$ for every $t \in(0, \infty) \backslash \mathcal{N}$.

(ii) $\int_{0}^{\infty} \mathbb{E}\left[G\left(t-\bar{S}_{r}\right) 1_{\left\{t \geq \bar{S}_{r}\right\}}\right] d r=t$ for every $t>0$.

(iii) $\int_{0}^{\infty} \mathbb{E}\left[G\left(t-S_{r}\right) 1_{\left\{t \geq S_{r}\right\}}\right] d r \leq t$ for every $t>0$.

Proof. (i) just follows from Lemma 2.1 by taking $s \rightarrow \infty$.

(ii) For $t>0$, we have by (i) and Fubini theorem that

$$
t=\int_{0}^{t}\left(\int_{0}^{\infty} \mathbb{E}\left[w\left(s-\bar{S}_{r}\right) 1_{\left\{s \geq \bar{S}_{r}\right\}}\right] d r\right) d s
$$




$$
\begin{aligned}
& =\int_{0}^{\infty} \mathbb{E}\left[\int_{0}^{t} w\left(s-\bar{S}_{r}\right) 1_{\left\{s \geq \bar{S}_{r}\right\}} d s\right] d r \\
& =\int_{0}^{\infty} \mathbb{E}\left[G\left(t-\bar{S}_{r}\right) 1_{\left\{t \geq \bar{S}_{r}\right\}}\right] d r .
\end{aligned}
$$

(iii) Since $G(x)$ is an increasing function in $x$, we have by (ii)

$$
\int_{0}^{\infty} \mathbb{E}\left[G\left(t-S_{r}\right) 1_{\left\{t \geq S_{r}\right\}}\right] d r \leq \int_{0}^{\infty} \mathbb{E}\left[G\left(t-\bar{S}_{r}\right) 1_{\left\{t \geq \bar{S}_{r}\right\}}\right] d r=t .
$$

This proves the corollary.

We define the generalized Caputo derivative $\partial_{t}^{w}$ by

$$
\partial_{t}^{w} f(t):=\frac{d}{d t} \int_{0}^{t} w(t-s)(f(s)-f(0)) d s
$$

whenever it is well-defined in some function space of $f$.

Suppose that $\left\{T_{t} ; t \geq 0\right\}$ is a strongly continuous semigroup with infinitesimal generator $(\mathcal{L}, \mathcal{D}(\mathcal{L}))$ in some Banach space $(\mathbb{B},\|\cdot\|)$ with the property that $\sup _{t>0}\left\|T_{t}\right\|<\infty$. Here $\left\|T_{t}\right\|$ denotes the operator norm of the linear map $T_{t}: \mathbb{B} \rightarrow \mathbb{B}$. Note that by the uniform boundedness principle, $\sup _{t>0}\left\|T_{t}\right\|<\infty$ is equivalent to $\sup _{t>0}\left\|T_{t} f\right\|<\infty$ for every $f \in \mathbb{B}$. Typical examples of such uniformly bounded strongly continuous semigroups are:

(i) Transition semigroup $\left\{P_{t} ; t \geq 0\right\}$ of a strong Markov process $X=\left\{X_{t}, t \geq 0 ; \mathbb{P}_{x}, x \in E\right\}$ on a Lusin space $E$ that has a weak dual with respect to some reference measure $m$ on $E$. Then for every $p \geq 1,\left\{P_{t} ; t \geq 0\right\}$ is a strongly continuous semigroup in $\mathbb{B}:=L^{p}(E ; m)$ with $\sup _{t>0}\left\|P_{t}\right\|_{p \rightarrow p} \leq 1$. The infinitesimal generator $(\mathcal{L}, \mathcal{D}(\mathcal{L}))$ of $\left\{P_{t} ; t \geq 0\right\}$ in $L^{p}(E ; m)$ is called the $L^{p}$ generator of the Markov process $X$.

(ii) Transition semigroup $\left\{P_{t} ; t \geq 0\right\}$ of a Feller process $X=\left\{X_{t}, t \geq 0 ; \mathbb{P}_{x}, x \in E\right\}$ on a locally compact separable Hausdorff space $E$. In this case, $\left\{P_{t} ; t \geq 0\right\}$ is a strongly continuous semigroup in the space $\left(C_{\infty}(E),\|\cdot\|_{\infty}\right)$ of continuous functions on $E$ that vanish at infinity equipped with uniform norm. The infinitesimal generator $(\mathcal{L}, \mathcal{D}(\mathcal{L}))$ of $\left\{P_{t} ; t \geq 0\right\}$ in $\mathbb{B}:=$ $\left(C_{\infty}(E),\|\cdot\|_{\infty}\right)$ is called the Feller generator of $X$.

(iii) Certain Feynman-Kac semigroups (can be non-local Feynman-Kac semigroups or even generalized Feynman-Kac semigroups) in $L^{p}$-space or in $C_{\infty}(E)$ of a Hunt process $X$; cf. [4, 6].

For $\alpha>0$, let $G_{\alpha}:=\int_{0}^{\infty} e^{-\alpha t} T_{t} d t$ be the resolvent of the semigroup $\left\{T_{t} ; t \geq 0\right\}$ on Banach space $\mathbb{B}$. Then by the resolvent equation, $\mathcal{D}(\mathcal{L})=G_{\alpha}(\mathbb{B})=G_{1}(\mathbb{B})$, which is dense in the Banach space $(\mathbb{B},\|\cdot\|)$.

Let $E_{t}:=\inf \left\{s>0: S_{s}>t\right\}, t \geq 0$, be the inverse subordinator. Define

$$
u(t, x)=\mathbb{E}\left[T_{E_{t}} f(x)\right]=\int_{0}^{\infty} T_{s} f(x) d_{s} \mathbb{P}\left(E_{t} \leq s\right)=\int_{0}^{\infty} T_{s} f(x) d_{s} \mathbb{P}\left(S_{s} \geq t\right) .
$$

The following is the main result of this paper, which gives the existence and uniqueness of solutions to time fractional equation (2.8). Theorem 1.1 is its particular case, where $T_{t}$ is the transition semigroup of a strong Markov process $X$ given by $T_{t} f(x)=\mathbb{E}_{x}\left[f\left(X_{t}\right)\right]$. 
Theorem 2.3 Suppose that $(\mathcal{L}, \mathcal{D}(\mathcal{L}))$ is the infinitesimal generator of a uniformly bounded strongly continuous semigroup $\left\{T_{t} ; t \geq 0\right\}$ in a Banach space $(\mathbb{B},\|\cdot\|)$. For every $f \in \mathcal{D}(\mathcal{L}), u(t, x):=$ $\mathbb{E}\left[T_{E_{t}} f(x)\right]$ is a solution in $(\mathbb{B},\|\cdot\|)$ to

$$
\left(\kappa \partial_{t}+\partial_{t}^{w}\right) u(t, x)=\mathcal{L} u(t, x) \quad \text { with } u(0, x)=f(x)
$$

in the following sense:

(i) $\sup _{t>0}\|u(t, \cdot)\|<\infty, x \mapsto u(t, x)$ is in $\mathcal{D}(\mathcal{L})$ for each $t \geq 0$ with $\sup _{t \geq 0}\|\mathcal{L} u(t, \cdot)\|<\infty$, and both $t \mapsto u(t, \cdot)$ and $t \mapsto \mathcal{L} u(t, \cdot)$ are continuous in $(\mathbb{B},\|\cdot\|)$;

(ii) for every $t>0, I_{t}^{w}(u):=\int_{0}^{t} w(t-s)(u(s, x)-f(x)) d s$ is absolutely convergent in $(\mathbb{B},\|\cdot\|)$ and

$$
\lim _{\delta \rightarrow 0} \frac{1}{\delta}\left(\kappa(u(t+\delta, \cdot)-u(t, \cdot))+I_{t+\delta}^{w}(u)-I_{t}^{w}(u)\right)=\mathcal{L} u(t, x) \quad \text { in }(\mathbb{B},\|\cdot\|) .
$$

When $\kappa>0, t \mapsto u(t, \cdot)$ is globally Lipschitz continuous in $(\mathbb{B},\|\cdot\|)$ and hence $\partial_{t} u(t, \cdot)$ exists in $(\mathbb{B},\|\cdot\|)$ for a.e. $t \geq 0.1$

Conversely, if $u(t, x)$ is a solution to (2.8) in the sense of (i) and (ii) above with $f \in \mathcal{D}(\mathcal{L})$, then $u(t, x)=\mathbb{E}\left[T_{E_{t}} f(x)\right]$ in $\mathbb{B}$ for every $t \geq 0$.

Proof. (a) (Existence) Clearly for $f \in \mathcal{D}(\mathcal{L})$,

$$
\sup _{t>0}\|u(t, \cdot)\| \leq \sup _{t>0} \mathbb{E}\left[\left\|T_{E_{t}} f\right\|\right] \leq \sup _{r>0}\left\|T_{r} f\right\|<\infty .
$$

By the same reason, $\sup _{t>0} \mathbb{E}\left[\left\|T_{E_{t}} \mathcal{L} f\right\|\right] \leq \sup _{r>0}\left\|T_{r} \mathcal{L} f\right\|<\infty$. Since

$$
\left.\left.\lim _{\delta \rightarrow 0} \frac{1}{\delta}\left(T_{\delta} u(t, \cdot)-u(t . \cdot)\right)=\lim _{\delta \rightarrow 0} \mathbb{E}\left[T_{E_{t}}\left(T_{\delta} f-f\right) / \delta\right)\right)\right]=\mathbb{E}\left[T_{E_{t}} \mathcal{L} f\right]
$$

in $(\mathbb{B},\|\cdot\|)$. we conclude that $u(t, \cdot) \in \mathcal{D}(\mathcal{L})$ with $\mathcal{L} u(t, \cdot)=\mathbb{E}\left[T_{E_{t}} \mathcal{L} f\right]$ for every $t>0$. Since $\left\{T_{t} ; t \geq 0\right\}$ is a strongly continuous semigroup on $\mathbb{B}$ with $\sup _{t \geq 0}\left\|T_{t}\right\|<\infty$ and $t \mapsto E_{t}$ is continuous a.s., we have by bounded convergence theorem that both $t \mapsto u(t, \cdot)=\mathbb{E}\left[T_{E_{t}} f\right]$ and $t \mapsto \mathcal{L} u(t, \cdot)=$ $\mathbb{E}\left[T_{E_{t}}(\mathcal{L} f)\right]$ are continuous in $(\mathbb{B},\|\cdot\|)$.

It follows from (2.7), (2.5), and the integration by parts formula that for every $t>0$,

$$
\begin{aligned}
& \int_{0}^{t} w(t-r)(u(r, x)-u(0, x)) d r \\
= & \int_{0}^{t} w(t-r)\left(\int_{0}^{\infty}\left(T_{s} f(x)-f(x)\right) d_{s} \mathbb{P}\left(S_{s} \geq r\right)\right) d r \\
= & \int_{0}^{\infty}\left(T_{s} f(x)-f(x)\right) d_{s}\left(\int_{0}^{t} w(t-r) \mathbb{P}\left(S_{s}>r\right) d r\right) \\
= & -\int_{0}^{\infty}\left(T_{s} f(x)-f(x)\right) d_{s} \mathbb{E}\left[G\left(t-S_{s}\right) 1_{\left\{t \geq S_{s}\right\}}\right] \\
= & \int_{0}^{\infty} \mathbb{E}\left[G\left(t-S_{s}\right) 1_{\left\{t \geq S_{s}\right\}}\right] \mathcal{L} T_{s} f(x) d s .
\end{aligned}
$$

\footnotetext{
${ }^{1}$ See Section 4 for an improved statement.
} 
Note that since $\sup _{s>0}\left\|T_{s} f\right\|<\infty$ and $\sup _{s>0}\left\|\mathcal{L} T_{s} f\right\|=\sup _{s>0}\left\|T_{s} \mathcal{L} f\right\|<\infty$, by (2.2) and Corollary 2.2, all the integrals in above display are absolutely convergent in the Banach space $(\mathbb{B},\|\cdot\|)$, while the second equality is justified by the Riemann sum approximation of Stieltjes integrals, Fubini theorem and the dominated convergence theorem 2 On the other hand, $\mathbb{P}\left(S_{r} \geq s\right)=1$ when $s \leq \kappa r$, while for a.e. $s \in(\kappa r, \infty)$, we have by Lemma 2.1 that

$$
\mathbb{P}\left(S_{r} \geq s\right)=\mathbb{P}\left(\bar{S}_{r} \geq s-\kappa r\right)=\int_{0}^{r} \mathbb{E}\left[w\left(s-\kappa r-\bar{S}_{y}\right) 1_{\left\{s-\kappa r>\bar{S}_{y}\right\}}\right] d y .
$$

So for every $t>0$,

$$
\begin{aligned}
\int_{0}^{t} \mathbb{P}\left(S_{r} \geq s\right) d s & =(\kappa r) \wedge t+\mathbb{E} \int_{0}^{r}\left(\int_{(\kappa r) \wedge t}^{t} w\left(s-\kappa r-\bar{S}_{y}\right) 1_{\left\{s-\kappa r>\bar{S}_{y}\right\}} d s\right) d y \\
& =(\kappa r) \wedge t+1_{\{\kappa r<t\}} \mathbb{E} \int_{0}^{r} G\left(t-\kappa r-\bar{S}_{y}\right) 1_{\left\{t-\kappa r>\bar{S}_{y}\right\}} d y .
\end{aligned}
$$

Since

$$
\begin{aligned}
\mathcal{L} u(s, x) & =\mathcal{L} \mathbb{E}\left[T_{E_{s}} f(x)\right]=\mathbb{E}\left[T_{E_{s}} \mathcal{L} f(x)\right] \\
& =\int_{0}^{\infty} T_{r} \mathcal{L} f(x) d_{r} \mathbb{P}\left(E_{s} \leq r\right)=\int_{0}^{\infty} T_{r} \mathcal{L} f(x) d_{r} \mathbb{P}\left(S_{r} \geq s\right),
\end{aligned}
$$

we have by (2.9) and (2.10) that

$$
\begin{aligned}
& \int_{0}^{t} \mathcal{L} u(s, x) d s \\
= & \int_{0}^{t}\left(\int_{0}^{\infty} T_{r} \mathcal{L} f(x) d_{r} \mathbb{P}\left(S_{r} \geq s\right)\right) d s \\
= & \int_{0}^{\infty} T_{r} \mathcal{L} f(x) d_{r}\left(\int_{0}^{t} \mathbb{P}\left(S_{r} \geq s\right) d s\right) \\
= & \mathbb{E} \int_{0}^{t / \kappa} T_{r} \mathcal{L} f(x)\left(\kappa+G\left(t-\kappa r-\bar{S}_{r}\right) 1_{\left\{t-\kappa r>\bar{S}_{r}\right\}}-\kappa \int_{0}^{r} w\left(t-\kappa r-\bar{S}_{y}\right) 1_{\left\{t-\kappa r>\bar{S}_{y}\right\}} d y\right) d r \\
= & \int_{0}^{\infty} T_{r} \mathcal{L} f(x) \mathbb{E}\left[G\left(t-S_{r}\right) 1_{\left\{t \geq S_{r}\right\}}\right] d r+\kappa \int_{0}^{t / \kappa} T_{r} \mathcal{L} f(x)\left(1-\mathbb{P}\left(S_{r} \geq t\right)\right) d r
\end{aligned}
$$

\footnotetext{
${ }^{2}$ Since $\left\|\int_{0}^{\infty}\left(T_{s} f-f\right) d_{s} \mathbb{P}\left(S_{s}>r\right)\right\| \leq(M+1)\|f\|$ and $\int_{0}^{t} w(t-r) d r=\int_{0}^{t} w(s) d s<\infty$, by Riemann sum approximation, the dominated convergence theorem and Fubini's theorem, for partitions $\Pi$ of $[0, \infty)$,

$$
\begin{aligned}
& \int_{0}^{t} w(t-r)\left(\int_{0}^{\infty}\left(T_{s} f(x)-f(x)\right) d_{s} \mathbb{P}\left(S_{s} \geq r\right)\right) d r \\
= & \int_{0}^{t} w(t-r) \lim _{\|\Pi\| \rightarrow 0} \sum_{i}\left(T_{s_{i}} f(x)-f(x)\right)\left(\mathbb{P}\left(S_{s_{i+1}}>r\right)-\mathbb{P}\left(S_{s_{i}}>r\right)\right) d r \\
= & \lim _{\|\Pi\| \rightarrow 0} \int_{0}^{t} w(t-r) \sum_{i}\left(T_{s_{i}} f(x)-f(x)\right)\left(\mathbb{P}\left(S_{s_{i+1}}>r\right)-\mathbb{P}\left(S_{s_{i}}>r\right)\right) d r \\
= & \left.\lim _{\|\Pi\| \rightarrow 0} \sum_{i}\left(T_{s_{i}} f(x)-f(x)\right)\left(\int_{0}^{t} w(t-r) \mathbb{P}\left(S_{s_{i+1}}>r\right) d r-\int_{0}^{t} w(t-r) \mathbb{P}\left(S_{s_{i}}>r\right)\right) d r\right) \\
= & \int_{0}^{\infty}\left(T_{s} f(x)-f(x)\right) d_{s}\left(\int_{0}^{t} w(t-r) \mathbb{P}\left(S_{s}>r\right) d r\right) .
\end{aligned}
$$
}




$$
\begin{aligned}
& =\int_{0}^{\infty} T_{r} \mathcal{L} f(x) \mathbb{E}\left[G\left(t-S_{r}\right) 1_{\left\{t \geq S_{r}\right\}}\right] d r+\kappa \int_{0}^{\infty} \mathbb{P}\left(S_{r}<t\right) d_{r}\left(T_{r} f(x)-f(x)\right) \\
& =\int_{0}^{\infty} T_{r} \mathcal{L} f(x) \mathbb{E}\left[G\left(t-S_{r}\right) 1_{\left\{t \geq S_{r}\right\}}\right] d r+\kappa \int_{0}^{t / \kappa} \mathbb{P}\left(E_{t}>r\right) d_{r}\left(T_{r} f(x)-f(x)\right) \\
& =\int_{0}^{\infty} T_{r} \mathcal{L} f(x) \mathbb{E}\left[G\left(t-S_{r}\right) 1_{\left\{t \geq S_{r}\right\}}\right] d r+\kappa \int_{0}^{\infty}\left(T_{r} f(x)-f(x)\right) d_{r} \mathbb{P}\left(E_{t} \leq r\right) \\
& =\int_{0}^{\infty} T_{r} \mathcal{L} f(x) \mathbb{E}\left[G\left(t-S_{r}\right) 1_{\left\{t \geq S_{r}\right\}}\right] d r+\kappa \mathbb{E}\left[T_{E_{t}} f(x)-f(x)\right] \\
& =\int_{0}^{\infty} T_{r} \mathcal{L} f(x) \mathbb{E}\left[G\left(t-S_{r}\right) 1_{\left\{t \geq S_{r}\right\}}\right] d r+\kappa(u(t, x)-u(0, x)) .
\end{aligned}
$$

Thus we have for every $t>0$,

$$
\kappa(u(t, x)-u(0, x))+\int_{0}^{t} w(t-r)(u(r, x)-u(0, x)) d r=\int_{0}^{t} \mathcal{L} u(s, x) d s .
$$

Consequently, $\left(\kappa \partial_{t}+\partial_{t}^{w}\right) u(t, x)=\mathcal{L} u(t, x)$ in $\mathbb{B}$ as $t \mapsto \mathcal{L} u(t, \cdot)$ is continuous in $(\mathbb{B},\|\cdot\|)$.

Since $\left\{T_{t} ; t \geq 0\right\}$ is a uniformly bounded strongly continuous semigroup in $(\mathbb{B},\|\cdot\|)$, for $f \in \mathcal{D}(\mathcal{L})$ and $t_{2}>t_{1} \geq 0$

$$
\left\|T_{t_{2}} f-T_{t_{1}} f\right\| \leq \int_{t_{1}}^{t_{2}}\left\|\partial_{s} T_{s} f\right\| d s=\int_{t_{1}}^{t_{2}}\left\|\mathcal{L} T_{s} f\right\| d s=\int_{t_{1}}^{t_{2}}\left\|T_{s} \mathcal{L} f\right\| d s \leq c\|\mathcal{L} f\|\left|t_{2}-t_{1}\right| .
$$

Note that when $\kappa>0,\left|E_{t}-E_{s}\right| \leq|t-s| / \kappa$. Hence we have from the above display that for every $t>s \geq 0$,

$$
\|u(t, \cdot)-u(s, \cdot)\|=\left\|\mathbb{E}\left[T_{E_{t}} f-T_{E_{s}} f\right]\right\| \leq c_{1} \mathbb{E}\left|E_{t}-E_{s}\right| \leq c_{2}(t-s) ;
$$

that is, $t \mapsto u(t, \cdot)$ is globally Lipschitz continuous in $(\mathbb{B},\|\cdot\|)$. This implies in particular that $u(t, \cdot)$ is differentiable in $t$ as an element in $(\mathbb{B},\|\cdot\|)$ for a.e. $t>0$.

(b) (Uniqueness) Suppose that $u(t, x)$ is a solution to (2.8) in the sense of (i) and (ii) with $f \in \mathcal{D}(\mathcal{L})$. Then $v(t, x):=u(t, x)-\mathbb{E}\left[T_{E_{t}} f(x)\right]$ is a solution to (2.8) with $v(0, x)=0$. Hence we have for every $t>0$,

$$
\kappa v(t, x)+\int_{0}^{t} w(t-r) v(r, x) d r=\int_{0}^{t} \mathcal{L} v(s, x) d s .
$$

Let $V(\lambda, x):=\int_{0}^{\infty} e^{-\lambda t} v(t, x) d t, \lambda>0$, be the Laplace transform of $t \mapsto v(t, x)$. Clearly for every $\lambda>0, V(\lambda, \cdot) \in \mathbb{B}$ with $\|V(\lambda, \cdot)\| \leq \lambda^{-1} \sup _{t>0}\|v(t, \cdot)\|$. Since $v(t, \cdot) \in \mathcal{D}(\mathcal{L})$ for every $t>0$ with $\sup _{t>0}\|\mathcal{L} v(t, \cdot)\|<\infty$, we have by dominated convergence theorem that for every $\lambda>0$,

$$
\begin{aligned}
\lim _{\delta \rightarrow 0} \frac{1}{\delta}\left(T_{\delta} V(\lambda, \cdot)-V(\lambda, \cdot)\right) & =\lim _{\delta \rightarrow 0} \int_{0}^{\infty} e^{-\lambda t} \frac{1}{\delta}\left(T_{\delta} v(t, \cdot)-v(t, \cdot)\right) d t \\
& =\lim _{\delta \rightarrow 0} \int_{0}^{\infty} e^{-\lambda t}\left(\frac{1}{\delta} \int_{0}^{\delta} T_{s} \mathcal{L} v(t, \cdot) d s\right) d t \\
& =\int_{0}^{\infty} e^{-\lambda t} \mathcal{L} v(t, \cdot) d t
\end{aligned}
$$

This shows that for each $\lambda>0, V(\lambda, \cdot) \in \mathcal{D}(\mathcal{L})$ with

$$
\mathcal{L} V(\lambda, \cdot)=\int_{0}^{\infty} e^{-\lambda t} \mathcal{L} v(t, \cdot) d t \quad \text { and } \quad\|\mathcal{L} V(\lambda, \cdot)\| \leq \int_{0}^{\infty} e^{-\lambda t}\|\mathcal{L} v(t, \cdot)\| d t \leq \frac{1}{\lambda} \sup _{t>0}\|\mathcal{L} v(t, \cdot)\| .
$$


Taking Laplace transform in $t$ on both sides of (2.11) yields

$$
V(\lambda, x)\left(\kappa+\int_{0}^{\infty} e^{-\lambda s} w(s) d s\right)=\frac{1}{\lambda} \int_{0}^{\infty} e^{-\lambda t} \mathcal{L} v(t, x) d t=\frac{\mathcal{L} V(\lambda, x)}{\lambda} .
$$

Thus by (2.1) and (2.3) $), \mathcal{L} V(\lambda, x)=\left(\kappa \lambda+\phi_{0}(\lambda)\right) V(\lambda, x)=\phi(\lambda) V(\lambda, x)$. In other words,

$$
(\phi(\lambda)-\mathcal{L}) V(\lambda, x)=0 \quad \text { for every } \lambda>0 .
$$

Since $\mathcal{L}$ is the infinitesimal generator of a uniformly bounded strongly continuous semigroup $\left\{T_{t}, t \geq\right.$ $0\}$ in Banach space $\mathbb{B}$, for every $\alpha>0$, the resolvent $G_{\alpha}=\int_{0}^{\infty} e^{-\alpha t} T_{t} d t$ is well defined and is the inverse to $\alpha-\mathcal{L}$. Hence we have from the last display that $V(\lambda, \cdot)=0$ in $\mathbb{B}$ for every $\lambda>0$. By the uniqueness of Laplace transform, we have $v(t, \cdot)=0$ in $\mathbb{B}$ for every $t>0$. This establishes that $u(t, x)=\mathbb{E}\left[T_{E_{t}} f(x)\right]$ in $\mathbb{B}$ for every $t \geq 0$.

Remark 2.4 (i) The assumption that $f \in \mathcal{D}(\mathcal{L})$ in Theorem 2.3 is to ensure that all the integrals involved in the proof of Theorem 2.3 are absolutely convergent in the Banach space $\mathbb{B}$. This condition can be relaxed if we formulate the equation (2.8) in the weak sense when the uniformly bounded strongly continuous semigroup $\left\{T_{t} ; t \geq 0\right\}$ is symmetric in a Hilbert space $L^{2}(E ; m)$ and so its quadratic form can be used to formulate weak solutions. This will be carried out in the ongoing joint work [5] with Kim, Kumagai and Wang. It in particular applies to the case where $\left\{T_{t} ; t \geq 0\right\}$ is the transition semigroup of any $m$-symmetric Markov process on a Lusin space $E$, which is a strongly continuous contraction symmetric semigroup in $L^{2}(E ; m)$.

(ii) There are two closely related work [19, 12]. Suppose that $X=\left\{X_{t}, t \geq 0 ; \mathbb{P}_{x}, x \in \mathbb{R}^{d}\right\}$ is a Lévy process on $\mathbb{R}^{d}$ and generator $\mathcal{L}$, and $S=\left\{S_{t} ; t \geq 0\right\}$ is a driftless subordinator with Laplace exponent $\phi$ and Lévy measure $\mu$. Let $E_{t}:=\inf \left\{s>0: S_{s}>t\right\}$ be the inverse subordinator. Under the assumption that $\kappa=0, \mu(0, \infty)=\infty, \int_{0}^{1} x|\log x| \mu(d x)<\infty$ and that the Lévy process $X$ has a transition density function, it is shown in [19, Theorem 4.1] that $u(t, x):=\mathbb{E}_{x}\left[f\left(X_{E_{t}}\right)\right]$ is a mild solution of the following pseudo-differential equation

$$
\phi\left(\partial_{t}\right) u(t, x)=\mathcal{L} u(t, x)+f(x) \mu(t, \infty) .
$$

Here $\phi\left(\partial_{t}\right)$ is a pseudo-differential operator in time variable $t$ formulated using Fourier multiplier.

Under the assumption that the Lévy measure $\mu$ of the subordinator $S_{t}$ satisfying condition $\mu(d \xi) \geq \xi^{1+\beta} d \xi$ on $(0, \varepsilon)$ for some $\varepsilon>0$ and $\beta>0$, and $\left\{T_{t} ; t \geq 0\right\}$ is the transition semigroup of a Feller process $X=\left\{X_{t}, t \geq 0 ; \mathbb{P}_{x}, x \in \mathbb{R}^{d}\right\}$ on $\mathbb{R}^{d}$ whose domain of infinitesimal generator contains $C^{2}\left(\mathbb{R}^{d}\right) \cap C_{\infty}\left(\mathbb{R}^{d}\right)$, [12, Theorem 8.4.2] asserts that for every $f \in C^{2}\left(\mathbb{R}^{d}\right) \cap C_{\infty}\left(\mathbb{R}^{d}\right)$, $u(t, x):=\mathbb{E}_{x}\left[f\left(X_{E_{t}}\right)\right]$ satisfies

$$
A_{t}^{*} u(t, x)=\mathcal{L} u(t, x)+f(x) A^{*}\left(1_{(0, \infty)}\right)(t) \quad \text { with } u(0, x)=f(x),
$$

where $A^{*}$ is the dual of the infinitesimal generator of the subordinator $S_{t}$ and notation $A_{t}^{*} u(t, x)$ means that the operator $A^{*}$ is applied to the function $t \mapsto u(t, x)$. Here $C^{2}\left(\mathbb{R}^{d}\right)$ is 
the space of $C^{2}$-smooth functions on $\mathbb{R}^{d}$ and $C_{\infty}\left(\mathbb{R}^{d}\right)$ is the space of continuous functions on $\mathbb{R}^{d}$ that vanish at infinity. In [12, Theorem 8.4.2], the subordinator $S_{t}$ may have drift $\kappa \geq 0$.

Similar problem has also been considered in [24] under more restrictive conditions and using a different approach. The time fractional derivative there is of the form

$$
\kappa \frac{\partial u(t)}{\partial t}+\int_{0}^{t} w(s) \frac{\partial}{\partial t} u(t-s) d s .
$$

This requires regularity assumption beyond absolute continuity on the function $t \mapsto u(t)$, as $w(s)$ is unbounded near $s=0$. The absolute convergence of the singular integral should be checked and justified.

(iii) Suppose the subordinator $S$ is driftless and has Lévy measure $\mu(d x)=\left(\int_{0}^{1} \frac{\beta}{x^{1+\beta}} \frac{c(\beta)}{\Gamma(1-\beta)} d \beta\right) d x$, where $c(\beta) \geq 0$ is a measurable function with $\int_{0}^{1} c(\beta) d \beta<\infty$. (Note that $\Gamma(1-\beta) \asymp \frac{1}{1-\beta}$ for $0<\beta<1$.) Then $w(x):=\mu(x, \infty)=\int_{0}^{1} x^{-\beta} \frac{c(\beta)}{\Gamma(1-\beta)} d \beta$. The time fractional derivative $\partial_{t}^{w}$ defined in this paper is the distributed-order fractional derivative defined in [18]. In this case, for continuously differentiable function $f$ on $[0, \infty)$, the time fractional derivative $\partial_{t}^{w} f(t)$ is the mixture of Caputo derivatives of order $\beta$ 's:

$$
\partial_{t}^{w} f(t)=\int_{0}^{1} \partial_{t}^{\beta} f(t) c(\beta) d \beta
$$

(iv) Cauchy problems with distributed order time fractional derivatives (where $\kappa=0$ ) were also studied in [16] for uniformly elliptic generators of divergence form in bounded $C^{1, \gamma}$ domains with Dirichlet boundary condition, under certain regularity conditions of the diffusion matrices. We also mention [14, Theorem 2] where $\left\{S_{t} ; \geq 0\right\}$ is a subordinator without drift and $\left\{T_{t} ; t \geq 0\right\}$ is the transition semigroup of a one-dimensional diffusion killed at certain rate via Feynman-Kac transform.

(v) There are limited results in literature on the uniqueness for the time fractional equations (2.8); see [10, 11, 15] for cases of $\partial_{t}^{\beta} u=\mathcal{L} u$ and [13] for distributed order time fractional equation $\partial_{t}^{w} u=\mathcal{L} u$ where $\mathcal{L}$ is a one-dimensional differential operator in a bounded interval. We mention that Remark 3.1 of a recent preprint [1] contains a uniqueness result for solutions to $\partial_{t}^{\beta} u=\mathcal{L} u$, where $\mathcal{L}$ is the Feller generator of a doubly Feller process killed upon leaving a bounded regular domain, proved also by using Laplace transform similar to our uniqueness proof for Theorem 2.3 in this paper.

(vi) When the uniformly bounded strongly continuous semigroup $\left\{T_{t} ; t \geq 0\right\}$ in Theorem 2.3 has an integral kernel $p(t, x, y)$ with respect to some measure $m(d x)$, then there is a kernel $q(t, x, y)$ so that

$$
u(t, x):=\mathbb{E}\left[T_{E_{t}} f(x)\right]=\int_{E} q(t, x, y) f(y) m(d y) ;
$$

in other words,

$$
q(t, x, y):=\mathbb{E}\left[p\left(E_{t}, x, y\right)\right]=\int_{0}^{\infty} p(s, x, y) d_{s} \mathbb{P}\left(E_{t} \leq s\right)
$$

is the fundamental solution to the time fractional equation $\left(\kappa \partial_{t}+\partial_{t}^{w}\right) u=\mathcal{L} u$ under the setting of this paper. In [5], two-sided estimates on $q(t, x, y)$ are obtained when $\kappa=0$ and 
$\left\{T_{t} ; t \geq 0\right\}$ is the transition semigroup of a diffusion process that satisfies two-sided Gaussiantype estimates or of a stable-like process on metric measure spaces.

Example 2.5 (i) When $\left\{S_{t} ; t \geq 0\right\}$ is a $\beta$-stable subordinator with $0<\beta<1$ with Laplace exponent $\phi(\lambda)=\lambda^{\beta}$, it is easy to check that $S_{t}$ has no drift (i.e. $\kappa=0$ ) and its Lévy measure is $\mu(d x)=\frac{\beta}{\Gamma(1-\beta)} x^{-(1+\beta)} d x$. Hence

$$
w(x):=\mu(x, \infty)=\int_{x}^{\infty} \frac{\beta}{\Gamma(1-\beta)} y^{-(1+\beta)} d y=\frac{x^{-\beta}}{\Gamma(1-\beta)} .
$$

Thus the time fractional derivative $\partial_{t}^{w} f$ defined by (1.2) is exactly the Caputo derivative of order $\beta$ defined by (1.1). In this case, Theorem 2.3 recovers the main result of [2] and [17, Theorem 5.1].

(ii) We call a subordinator $\left\{S_{t} ; t \geq 0\right\}$ truncated $\beta$-stable subordinator if it is driftless and its Lévy measure is

$$
\mu_{\delta}(d x)=\frac{\beta}{\Gamma(1-\beta)} x^{-(1+\beta)} 1_{(0, \delta]}(x) d x
$$

for some $\delta>0$. In this case,

$$
w_{\delta}(x):=\mu_{\delta}(x, \infty)=1_{\{0<x \leq \delta\}} \int_{x}^{\delta} \frac{\beta}{\Gamma(1-\beta)} y^{-(1+\beta)} d y=\frac{1}{\Gamma(1-\beta)}\left(x^{-\beta}-\delta^{-\beta}\right) 1_{(0, \delta]}(x) .
$$

So the corresponding the fractional derivative of $(\underline{1.2})$ is

$$
\partial_{t}^{w_{\delta}} f(t):=\frac{1}{\Gamma(1-\beta)} \frac{d}{d t} \int_{(t-\delta)^{+}}^{t}\left((t-s)^{-\beta}-\delta^{-\beta}\right)(f(s)-f(0)) d s .
$$

This is the fractional-time derivative whose value at time $t$ depends only on the $\delta$-range of the past of $f$ as mentioned in the Introduction. Theorem 2.3 says that the corresponding time fractional equation (1.5) can be solved by using the inverse of truncated $\beta$-stable subordinator. Clearly, as $\lim _{\delta \rightarrow \infty} w_{\delta}(x)=w(x):=\frac{1}{\Gamma(1-\beta)} x^{-\beta}$. Consequently, the fractional derivative $\left.\partial_{t}^{w_{\delta}} f(t) \rightarrow \partial_{t}^{w} f(t)\right)$, the Caputo derivative of $f$ of order $\beta$, in the distributional sense as $\delta \rightarrow 0$. Using the probabilistic representation in Theorem 2.3. one can deduce that as $\delta \rightarrow \infty$, the solution to the equation $\partial_{t}^{w_{\delta}} u=\mathcal{L} u$ with $u(0, x)=f(x)$ converges to the solution of $\partial_{t}^{\beta} u=\mathcal{L} u$ with $u(0, x)=f(x)$.

If we define

$$
\eta_{\delta}(r)=\frac{\Gamma(2-\beta) \delta^{\beta-1}}{\beta} w_{\delta}(r)=(1-\beta) \delta^{\beta-1}\left(x^{-\beta}-\delta^{-\beta}\right) 1_{(0, \delta]}(x),
$$

then $\eta_{\delta}(r)$ converges weakly to the Dirac measure concentrated at 0 as $\delta \rightarrow 0$. So the fractional derivative $\partial_{t}^{\eta_{\delta}} f(t)$ converges to $f^{\prime}(t)$ for every differentiable $f$. It can be shown that the subordinator corresponding to $\eta_{\delta}$, that is, subordinator with Lévy measure

$$
\nu_{\delta}(d x):=\frac{(1-\beta) \delta^{\beta-1}}{\beta} x^{-(1+\beta)} 1_{(0, \delta]}(x) d x,
$$

converges as $\delta \rightarrow 0$ to deterministic motion $t$ moving at constant speed 1 . Using Theorem 2.3. one can show that the solution to the equation $\partial_{t}^{\eta_{\delta}} u(t, x)=\mathcal{L} u(t, x)$ with $u(0, x)=f(x)$ converges to the solution of the heat equation $\partial_{t} u=\mathcal{L} u$ with $u(0, x)=f(x)$. 


\section{Occupation measure for processes time-changed by inverse sub- ordinator}

Suppose $X=\left\{X_{t}, t \geq 0 ; \mathbb{P}_{x}, x \in E\right\}$ is a general strong Markov process on state space $E$ with infinitesimal generator $\mathcal{L}$, and $S=\left\{S_{t} ; t \geq 0\right\}$ is a subordinator independent of $X$ whose Lévy measure $\mu$ satisfies $\mu(0, \infty)=\infty$. Let $\phi$ be the Laplace exponent of $S$; that is, $\mathbb{E} e^{-\lambda S_{t}}=e^{-t \phi(\lambda)}$. Note that $\mathbb{E}\left[S_{t}\right]=t \phi^{\prime}(0)$ so in particular $\phi^{\prime}(0)=\mathbb{E}\left[S_{1}\right]$. Let $E_{t}:=\inf \left\{s>0: S_{s}>t\right\}$ be the inverse subordinator, and $X_{t}^{*}:=X_{E_{t}}$. Suppose $D$ is an open subset of $E$ and define $\tau_{D}:=\left\{t>0: X_{t} \notin D\right\}$ to be the first exit time from $D$ by the process $X$. In general, the time-changed process $X^{*}$ is not a Markov process but we can still define its first exit time from $D$ by

$$
\tau_{D}^{*}:=\inf \left\{t>0: X_{t}^{*} \notin D\right\}
$$

Let $\partial$ be a cemetery point. The process $X^{*, D}$ defined by $X_{t}^{*, D}:=X_{t}^{*}$ when $t<\tau_{D}^{*}$ and $X_{t}^{*}:=\partial$ for $t \geq \tau_{D}^{*}$ is called the part process of $X^{*}$ in $D$. The part process $X^{D}$ of $X$ in $D$ is defined in an analogous way. We use $\mathbb{E}_{x}$ to denote mathematical expectation taken with respect to the probability law $\mathbb{P}_{x}$, under which the Markov process $X$ starts from $x \in E$. For every $x \in D$, the occupation measures for $X^{D}$ and $X^{*, D}$ are defined by

$$
\nu_{x}^{D}(A)=\mathbb{E}_{x}\left[\int_{0}^{\tau_{D}} 1_{A}\left(X_{s}\right) d s\right] \quad \text { and } \quad \nu_{x}^{*, D}(A)=\mathbb{E}_{x}\left[\int_{0}^{\tau_{D}^{*}} 1_{A}\left(X_{s}^{*}\right) d s\right], \quad A \subset D .
$$

Occupation measures describe the average amount of time spent by the processes in subsets of the state space.

The next theorem says that the occupation measure for the part process $X^{*, D}$ of $X^{*}$ in $D$ is proportional to that of the part process $X^{D}$ of $X$ in $D$ when $\phi^{\prime}(0)<\infty$, that is, when the subordinator $S_{t}$ has finite mean. When the subordination $S_{t}$ has infinite mean, the occupation measure for the part process $X^{*, D}$ of $X^{*}$ in $D$ is always infinite.

Theorem 3.1 For every measurable function $f \geq 0$ on $D$ and $x \in D$,

$$
\mathbb{E}_{x}\left[\int_{0}^{\tau_{D}^{*}} f\left(X_{t}^{*}\right) d t\right]=\phi^{\prime}(0) \mathbb{E}_{x}\left[\int_{0}^{\tau_{D}} f\left(X_{t}\right) d t\right]=\phi^{\prime}(0) G_{D} f(x) .
$$

In other words, $\nu_{x}^{*, D}=\phi^{\prime}(0) \nu_{x}^{D}$ for every open set $D \subset E$ and every $x \in D$.

Proof. First note that

$$
\begin{aligned}
\tau_{D}^{*} & =\inf \left\{t>0: X_{E_{t}} \notin D\right\}=\inf \left\{t>0: E_{t}=\tau_{D}\right\} \\
& =\inf \left\{t>0: S_{\tau_{D}}>t\right\}=S_{\tau_{D}} .
\end{aligned}
$$

For any $f \geq 0$ on $D$, we have using the independence between the strong Markov process $X$ and the subordinator $S$ that

$$
\mathbb{E}_{x}\left[\int_{0}^{\tau_{D}^{*}} f\left(X_{t}^{*}\right) d t\right]=\mathbb{E}_{x}\left[\int_{0}^{S_{\tau_{D}}} f\left(X_{E_{t}}\right) d t\right]=\mathbb{E}_{x}\left[\int_{0}^{\tau_{D}} f\left(X_{r}\right) d S_{r}\right]
$$




$$
\begin{aligned}
& =\mathbb{E}_{x}\left[\int_{0}^{S_{\tau_{D}}} f\left(X_{E_{t}}\right) d t\right]=\mathbb{E}_{x} \mathbb{E}_{x}\left[\int_{0}^{\tau_{D}} f\left(X_{r}\right) d S_{r} \mid X\right] \\
& =\mathbb{E}_{x}\left[\int_{0}^{\tau_{D}} f\left(X_{r}\right) d\left(\mathbb{E} S_{r}\right)\right]=\mathbb{E}_{x}\left[\int_{0}^{\tau_{D}} f\left(X_{r}\right) d r\right] \phi^{\prime}(0) \\
& =\phi^{\prime}(0) G_{D} f(x) .
\end{aligned}
$$

Remark 3.2 (i) Taking $f=1$ in Theorem 3.1 in particular yields the following relation on mean exit times:

$$
\mathbb{E}_{x}\left[\tau_{D}^{*}\right]=\phi^{\prime}(0) \mathbb{E}_{x}\left[\tau_{D}\right] \quad \text { for every } x \in D .
$$

When $X$ is either a diffusion process determined by a stochastic differential equation driven by Brownian motion or a rotationally symmetric $\alpha$-stable process on $\mathbb{R}^{d}$, and $\left\{S_{t} ; t \geq 0\right\}$ is a tempered $\beta$-stable subordinator having Laplace exponent $\phi(\lambda)=(\lambda+m)^{\beta}-m^{\beta}$ for some $m>0$ and $0<\beta<1$, (3.1) recovers the main result of [7], derived there using a PDE method.

(ii) Observe that the part process $X^{D}$ of $X$ killed upon leaving $D$ is a strong Markov process in $D$ whose infinitesimal generator $\mathcal{L}^{D}$ is $\mathcal{L}$ in $D$ having zero exterior condition. The transition semigroup of $X^{D}$ is $P_{t}^{D} f:=\mathbb{E}_{x}\left[f\left(X_{t}\right) ; t<\tau_{D}\right]$. Hence by Theorem 2.3, for $f \in \mathcal{D}\left(\mathcal{L}^{D}\right)$,

$$
u(t, x):=\mathbb{E}_{x}\left[f\left(X_{E_{t}}^{D}\right)\right]=\mathbb{E}_{x}\left[f\left(X_{t}^{*}\right) ; t<\tau_{D}^{*}\right]
$$

is the strong solution to

$$
\left(\kappa \partial_{t}+\partial_{t}^{w}\right) u(t, x)=\mathcal{L}^{D} u(t, x) \quad \text { with } u(0, x)=f(x) \text { in } D .
$$

On the other hand, $G_{D} f(x)$ is the solution to the Poisson equation $\mathcal{L} v=-f$ in $D$ with $v=0$ on $D^{c}$. Hence it follows from Theorem 3.1 that for $f \in \mathcal{D}\left(\mathcal{L}^{D}\right)$,

$$
G_{D}^{*} f(x)=\int_{0}^{\infty} \mathbb{E}_{x}\left[f\left(X_{t}\right) ; t<\tau_{D}^{*}\right] d t=\int_{0}^{t} u(t, x) d t
$$

is the solution to the Poisson equation

$$
\mathcal{L}^{D} v=-\phi^{\prime}(0) f \quad \text { in } D \quad \text { with } v=0 \text { on } D^{c} .
$$

Since by (ii) of Theorem 2.3 that

$$
\lim _{\delta \rightarrow 0} \frac{1}{\delta}\left(\kappa(u(t+\delta, \cdot)-u(t, \cdot))+I_{t+\delta}^{w}(u)-I_{t}^{w}(u)\right)=\mathcal{L} u(t, x) \quad \text { in }(\mathbb{B},\|\cdot\|) .
$$

and $t \rightarrow \mapsto \mathcal{L} u(t, \cdot)$ is continuous in $(\mathbb{B},\|\cdot\|)$, we conclude that

$$
\partial_{t} u(t, \cdot):=\lim _{\delta \rightarrow 0} \frac{1}{\delta}((u(t+\delta, \cdot)-u(t, \cdot)))=\mathcal{L} u(t, \cdot)-\partial_{t}^{w} u
$$

exists and $t \mapsto \partial_{t} u$ is continuous in $(\mathbb{B},\|\cdot\|)$.

Acknowledgement. The author thanks M. M. Meerschaert for the invitation to the Workshop "Future Directions in Fractional Calculus Research and Applications" held at Michigan State University, East Lansing, from October 17-21, 2016, and for helpful comments. He also thanks T. Kumagai and J. Wang for helpful comments. 


\section{Note added after publication}

The last sentence in the existence part of Theorem 2.3 can be strengthened as follows:

When $\kappa>0, t \mapsto u(t, \cdot)$ is globally Lipschitz continuous in $(\mathbb{B},\|\cdot\|)$, and both $\partial_{t} u(t, \cdot)$ and $\partial_{t}^{w} u(t, \cdot):=\frac{d}{d t} I_{t}^{w}(u)$ exists as a continuous function taking values in $(\mathbb{B},\|\cdot\|)$.

Proof. Recall that $\mu$ is the Lévy measure for the subordinator $S_{t}$ and $w(x):=\mu(x, \infty)$ for $x>0$. By (2.2) and the monotone convergence theorem,

$$
\lim _{r \rightarrow 0} r w(r) \leq \lim _{r \rightarrow 0} \int_{0}^{r} w(s) d s=0 .
$$

When $\kappa>0$, it is shown in Theorem 2.3 that $t \mapsto u(t, \cdot)$ is globally Lipschitz continuous in $(\mathbb{B},\|\cdot\|)$. So the following function taking values in $\mathbb{B}$ is well defined:

$$
g(s, \cdot):=(u(s, \cdot)-u(0, \cdot)) w(s)+\int_{(0, s]}(u(s, \cdot)-u(s-r, \cdot)) \mu(d r), \quad s>0,
$$

with $\lim _{s \rightarrow 0} g(s, \cdot)=0$ in $(\mathbb{B},\|\cdot\|) \cdot 3$ Moreover, denoting the global Lipschitz constant of $t \rightarrow u(t, \cdot)$ in $(\mathbb{B},\|\cdot\|)$ by $M_{1}$, we have for any $s>0$ and $\delta>0$,

$$
\begin{aligned}
\|g(s+\delta, \cdot)-g(s, \cdot)\| \leq & \|u(s+\delta, \cdot)-u(s, \cdot)\| w(s+\delta)+\|u(s,, \cdot)-u(0, \cdot)\||w(s+\delta)-w(s)| \\
& +\int_{(0, s]} \|(u(s+\delta, \cdot)-u(s+\delta-r, \cdot))-(u(s, \cdot)-u(s-r, \cdot) \| \mu(d r) \\
& +\int_{(s, s+\delta]}\|u(s+\delta, \cdot)-u(s+\delta-r, \cdot)\| \mu(d r) \\
\leq & M_{1} \delta w(s+\delta)+M_{1} s \mu(s, s+\delta]+M_{1} \int_{(s, s+\delta]} r \mu(d r) \\
& +\int_{(0, s]} \|(u(s+\delta, \cdot)-u(s+\delta-r, \cdot))-(u(s, \cdot)-u(s-r, \cdot) \| \mu(d r) .
\end{aligned}
$$

In view of (2.2) and (4.1), each of the first three terms converges to 0 as $\delta \rightarrow 0+$, and so does the fourth term by the dominated convergence theorem and the bound

$$
\|(u(s+\delta, \cdot)-u(s+\delta-r, \cdot))-\left(u(s, \cdot)-u(s-r, \cdot) \| \leq 2 M_{1} r .\right.
$$

Note that by definition (4.2),

$$
g(s, \cdot):=(u(s, \cdot)-u(0, \cdot)) w(s-)+\int_{(0, s)}(u(s, \cdot)-u(s-r, \cdot)) \mu(d r) \quad \text { for } s>0 .
$$

Using this expression, by a similar argument as above we have for every $s>0$,

$$
\lim _{\delta \rightarrow 0+}\|g(s-\delta, \cdot)-g(s, \cdot)\|
$$

\footnotetext{
${ }^{3}$ This definition is motivated by the fact that were $u(r, \cdot)$ differentiable in $r$, then
}

$$
g(s, \cdot)=\int_{0}^{s} w(s-r) d_{r}(u(r, \cdot)-u(s, \cdot))=\int_{0}^{s} w(s-r) d_{r} u(r, \cdot)
$$

by an integration by parts. 


$$
\begin{aligned}
\leq & \lim _{\delta \rightarrow 0+}\left(M_{1} \delta w(s)+M_{1} s \mu(s-\delta, s)+M_{1} \int_{(s-\delta, s)} r \mu(d r)\right) \\
& +\lim _{\delta \rightarrow 0+} \int_{0}^{s} 1_{(0, s-\delta]}(r) \|(u(s, \cdot)-u(s-r, \cdot))-(u(s-\delta, \cdot)-u(s-\delta-r, \cdot) \| \mu(d r) \\
= & 0 .
\end{aligned}
$$

This establishes the claim that $s \mapsto g(s, \cdot)$ is continuous in $(\mathbb{B},\|\cdot\|)$ on $[0, \infty)$ with $g(0, \cdot):=0$. For every $t>0$,

$$
\begin{aligned}
\int_{0}^{t} g(s, \cdot) d s= & \int_{0}^{t}(u(s, \cdot)-u(0, \cdot)) w(t-s) d s+\int_{0}^{t}(u(s, \cdot)-u(0, \cdot))(w(s)-w(t-s)) d s \\
& +\int_{0}^{t}\left(\int_{(0, s]}(u(s, \cdot)-u(s-r, \cdot)) \mu(d r)\right) d s \\
= & \int_{0}^{t}(u(s, \cdot)-u(0, \cdot)) w(t-s) d s+\int_{0}^{t}(u(s, \cdot)-u(t-s, \cdot)) w(s) d s \\
& +\int_{(0, t]}\left(\int_{r}^{t}(u(s, \cdot)-u(s-r, \cdot)) d s\right) \mu(d r) \quad \text { (by Fubini's theorem) } \\
= & \int_{0}^{t}(u(s, \cdot)-u(0, \cdot)) w(t-s) d s+\int_{0}^{t}(u(s, \cdot)-u(t-s, \cdot)) w(s) d s \\
& +\int_{(0, t]}^{r}\left(\int_{t-r}^{t} u(s, \cdot) d s-\int_{0}^{r} u(s, \cdot) d s\right) \mu(d r) \\
= & \int_{0}^{t}(u(s, \cdot)-u(0, \cdot)) w(t-s) d s+\int_{0}^{t}(u(s, \cdot)-u(t-s, \cdot)) w(s) d s d s \\
& +\int_{(0, t]}\left(\int_{0}^{r}(u(t-s, \cdot)-u(s, \cdot)) d s\right) \mu(d r) \\
= & \int_{0}^{t}(u(s, \cdot)-u(0, \cdot)) w(t-s) d s+\int_{0}^{t}(u(s, \cdot)-u(t-s, \cdot)) w(s) d s \\
& +\int_{0}^{t}\left(\int_{(s, t]}(u(t-s, \cdot)-u(s, \cdot)) \mu(d r)\right) d s \quad \text { (by Fubini's theorem) } \\
= & \int_{0}^{t}(u(s, \cdot)-u(0, \cdot)) w(t-s) d s+\int_{0}^{t}(u(s, \cdot)-u(t-s, \cdot)) w(s) d s \\
& +\int_{0}^{t}(u(t-s, \cdot)-u(s, \cdot))(w(s)-w(t)) d s \\
&
\end{aligned}
$$

Since $t \mapsto g(t, \cdot)$ is continuous in $(\mathbb{B},\|\cdot\|)$ over $[0, \infty), \partial_{t}^{w} u(t, \cdot):=\frac{d}{d t} I_{t}^{w}(u)$ exists for every $t>0$ and $t \mapsto \partial_{t}^{w} u=g(t, \cdot)$ is continuous in $(\mathbb{B},\|\cdot\|)$. Now it follows from (ii) of Theorem 2.3 that

$$
\partial_{t} u(t, \cdot):=\lim _{\delta \rightarrow 0} \frac{1}{\delta}((u(t+\delta, \cdot)-u(t, \cdot)))=\frac{1}{\kappa}\left(\mathcal{L} u(t, \cdot)-\partial_{t}^{w} u(t, \cdot)\right)=\frac{1}{\kappa}(\mathcal{L} u(t, \cdot)-g(t, \cdot))
$$

exists and $t \mapsto \partial_{t} u(t, \cdot)$ is continuous in $(\mathbb{B},\|\cdot\|)$. Hence $u(t, x):=\mathbb{E}\left[T_{E_{t}} f(x)\right]$ satisfies

$$
\left.\kappa \partial_{t} u(t, x)\right)+\partial_{t}^{w} u(t, x)=\mathcal{L} u(t, x)
$$


in the strong sense in the Banach space $(\mathbb{B},\|\cdot\|)$.

\section{References}

[1] B. Baeumer, T. Luks and M. M. Meerschaert, Space-time fractional Dirichlet problems. Preprint.

[2] B. Baeumer and M. M. Meerschaert, Stochastic solutions for fractional Cauchy problems. Fract. Calc. Appl. Anal. 4 (2001), 481-500.

[3] J. Bertoin, Lévy Processes. Cambridge Univ. Press, 1998.

[4] Z.-Q. Chen, P. J. Fitzsimmons, K. Kuwae and T.-S. Zhang, Perturbation of symmetric Markov processes. Probab. Theory Relat. Fields 140 (2008), 239-275.

[5] Z.-Q. Chen, P. Kim, T. Kumagai and J. Wang, Heat kernel estimates for solutions of general time fractional equations. In preparation.

[6] Z.-Q. Chen and R. Song, Conditional gauge theorem for non-local Feynman-Kac transforms. Probab. Theory Relat. Fields, 125 (2003), 45-72.

[7] W. Deng, X. Wu and W. Wang, Mean exit time and escape probability for the anomalous processes with the tempered power-law waiting times. To appear in Europhysics Letters.

[8] Q. Du, J. Yang and Z. Zhou, Analysis of a nonlocal-in-time parabolic equation. Disc. Cont. Dyn. Sys. B. 22 (2017), 339-368.

[9] R. Herrmann, Fractional Calculus: An Introduction for Pysicists, 2nd Edition. Singapore: World Scientific, 2011.

[10] A. N. Kochubei, The Cauchy problem for evolution equations of fractional order. (Russian); translated from Differentsial'nye Uravneniya 25 (1989), 1359-1368, 1468 Differential Equations 25 (1989), 967-974.

[11] A. N. Kochubei, Diffusion of fractional order. (Russian); translated from Differentsial'nye Uravneniya 26 (1990), 660-670, 733-734 Differential Equations 26 (1990), 485-492.

[12] V. Kolokoltsov, Markov Processes: Semigroups and Generators. De Gruyter, Berlin/New York, 2011.

[13] Y. Luchko, Boundary value problems for the generalized time-fractional diffusion equation of distributed order. Fract. Calc. Appl. Anal. 12 (2009), $409 ? 422$.

[14] M. Magdziarz and R. L. Schilling, Asymptotic properties of Brownian motion delayed by inverse subordinators. Proc. Amer. Math. Soc. 143 (2015), 4485-4501.

[15] M. M. Meerschaert, E. Nane and P. Vellaisamy, Fractional Cauchy problems on bounded domains. Ann. Probab. 37 (2009), 979-1007. 
[16] M. M. Meerschaert, E. Nane and P. Vellaisamy, Distributed-order fractional Cauchy problems on bounded domains. J. Math. Anal. Appl. 379 (2011), 216-228.

[17] M. M. Meerschaert and H. P. Scheffler, Limit theorems for continuous time random walks with infinite mean waiting times. J. Appl. Probab. 41 (2004), 623-638.

[18] M. M. Meerschaert and H. P. Scheffler, Stochastic model for ultraslow diffusion. Stochastic Process Appl. 116 (2006), 1215-1235.

[19] M. M. Meerschaert and H. P. Scheffler, Triangular array limits for continuous time random walks. Stochastic Process Appl. 118 (2008), 1606-1633.

[20] M. M. Meerschaert and A. Sikorskii, Stochastic Models for Fractional Calculus, De Gruyter Studies in Mathematics 43. Walter de Gruyter, Berlin/Boston, 2012.

[21] R. Metzler and J. Klafter, The restaurant at the end of the random walk: recent developments in the description of anomalous transport by fractional dynamics. J. Pyhsics A 37 (2004), R161-R208.

[22] R. Metzeler and J. Klafter, The random walk's guide to anomalous diffusion: a fractional dynamics approach. Phys. Peps. 339(1) (2000), 1-77.

[23] M. Shlesinger, J. Klafter and Y. M. Wong, Random walks with infinite spatial and temporal moments. J. Statist. Phys. 27 (1982), 499-512.

[24] B. Toaldo, Convolution-type derivatives, hitting-times of subordinators and time-changed $C_{0^{-}}$ semigroups. Potential Anal. 42 (2015), 115-140.

\section{Zhen-Qing Chen}

Department of Mathematics, University of Washington, Seattle, WA 98195, USA

E-mail: zqchen@uw.edu 